\title{
Building structure topology optimization
}

\author{
Xianjie WANG ${ }^{1, a}$, Yong SUN ${ }^{1, b}$, Mengjie XIANG ${ }^{1, c}$ \\ ${ }^{1}$ School of Architecture and Planning, Yunnan University, Kunming, 650091, China \\ axianjiewang@mail.nwpu.edu.cn, b751537272@qq.com, 'c1261767456 @qq.com
}

Keywords: Bi-directional Evolutionary Structural Optimization (BESO); Computer Program Development; Estimate Criterion; Building Structure; Optimization

\begin{abstract}
In this paper, a new estimate criterion is introduced to make the optimization process reasonable and credible, and it has a good control effect for the convergence of solution. An improved optimization program is exploiting for the directed building structure by means of a distinction design between the active and non-active elements. With the help of this plug-in program, the data can be exchanged between the FEA software of ABAQUS and the post-processor package in MATLAB. Several examples have shown the computer program can be used for directed structure topology optimization and portion construction design. This method is presented here for a wide used in structure design.
\end{abstract}

\section{Introduction}

In the traditional, structure design process is design - Analysis - check - redesign.[1] And this blind check is depending on the designer's experience, which is hard to get the optimal design scheme. Structure optimization design is aimed for the minimum volume, higher stiffness and suitable frequency by changing the size, shape and topology of the structure. Building structure topology optimization is one of the active research directions in the field of topology optimization, which is an initiative and well-regulated search process in order to achieve the predetermined optimal goal. [2]

Compared with other methods, the Bi-directional Evolutionary Structural Optimization (BESO) is convergent, clear boundary and good versatility, which is more and more widely used in engineering design.[3] However, it is hardly to get the desired results just by the stress or energy elimination criteria. [4] It is just because some unimportant components for the mechanical properties but with actual function must be consider existing and retained. Such special components optimization problem needs to be solved before practical engineering application.

To compensate for the imperfection of traditional BESO, the improved criterion and regional design are established. It is successfully used for the particular function or aesthetic requirement building structure, which is expanding the BESO method in practical engineering application.

\section{The improved BESO algorith}

To get the minimum mean compliance for structure with a given volume of material, the structure is optimized by elements removing and adding elements step by step. And, the optimization problem with the volume constraint can be stated as:

$\begin{cases}\min & C=\frac{1}{2} f^{T} u \\ \text { s.t. } & v^{*}-\sum_{i=1}^{N} v_{i} X_{i}=0 \quad X_{i}=X_{\min } \text { or } 1\end{cases}$

Here, $\mathrm{C}$ is the mean compliance. $f$ and $u$ are the applied load and displacement vectors. $\mathrm{N}$ is the total number of the solid elements. $\mathrm{V}^{*}$ and $\mathrm{v}_{\mathrm{i}}$ are the volume for the given structural and element, respectively. Here, $x_{\mathrm{i}}$ is the design variable, which sets as $x_{\min }$ and 1 in the system. 


\section{The filter function}

The balance equation for structural can be set as.

$K U=f$

Here, $\mathrm{K}$ is the structure stiffness matrix.

The stiffness change $(\Delta K)$ caused by the deletion of the i-th element from the structure is equivalent to the stiffness matrix of the i-th element, and can be expressed as follow.

$\Delta K=-K_{i}$

So, the structural displacement changed by the elements removed is:

$\Delta U=-K^{-1} \Delta K U$

The structural strain energy caused by the external loading can be changed into $\Delta C$ :

$\Delta C=\frac{1}{2} f^{T} \Delta U=\frac{1}{2} U_{i}^{T} K, U$,

Herein, $K_{i}$ and $U_{i}$ denote the elemental stiffness matrix and displacement matrix. It can be seen that the above sensitivity number can be got from the elemental strain energy. ${ }^{[5]}$

$\Delta \mathcal{C}_{i}=\left\{\begin{array}{cc}\frac{1}{2} U^{\prime} K U, & \text { for solid material } \\ 0 & \text { for soft material }\end{array}\right.$

To overcome the formation of checkerboard patterns, the sensitivity number is smoothed. ${ }^{[6]}$

Firstly, the nodal sensitivity numbers $\alpha_{i}^{n}$ are defined by averaging the elemental sensitivity numbers as follows.

$\alpha_{i}^{n}=\sum_{i=1}^{M} \omega_{i} \alpha_{i}^{e}$

Where $M$ denotes the total number of elements connected to the $\mathrm{j}$ th node. $\omega_{i}$ is the weight factor of the i th element can be defined by

$\omega_{i}=\frac{1}{M-2}\left(1-\frac{r_{i j}}{\sum_{i=1}^{M} r_{i j}}\right)$

Obviously, $\sum_{i=1}^{M} \omega_{i}=1$ 。 The more close to the node, the greater the influence of the element on the sensitivity of the node.

In order to ensure the convergence of the iterative process, the history of the iterative is averaged by the follow equation. ${ }^{[8]}$

$\alpha_{i}=\frac{\alpha_{i}^{n}+\alpha_{i}^{n-1}}{2}$

Where $\mathrm{n}$ is the current iteration number.

\section{The evolutionary criterion}

Based on the statistical principle, the discrete degree of data set can be assessment by the mean square deviation; the larger the data means more dispersed. So, the unit compliance variance PI, as a new BESO performance index, is introduced in this paper.

$P I=\sqrt{\frac{\sum_{i=1}^{N_{k}}\left(c_{i k}-\bar{C}_{k}\right)^{2}}{N_{k}}}$

Here, $N_{k}$ is the number of entities in the $\mathrm{K}$ iteration. $C_{i k}$ and $\bar{C}_{k}$ are respectively the strain energy of the i-th solid element and the average strain energy of all the solid elements in the $\mathrm{K}$ iteration. The smaller of PI, the more uniform the stress distribution of the structure is. 


\section{Building typical optimization problems}

An inverted L column design area shows in Figure 1. Fixed the bottom and concentrated load applied at the right side of the midpoint. The elastic modulus and density of the materials for steel are respectively $2.1 \mathrm{e} 11 \mathrm{GPa}$ and $7800 \mathrm{~kg} / \mathrm{m} 3$. The L column is modeled using 6400 four-node brick elements. The objective volume is only $18 \%$ of the design domain. BESO parameters are defined as: $\mathrm{ER}=2 \%$, rmin $=1.5$ and $\varepsilon=0.001$.

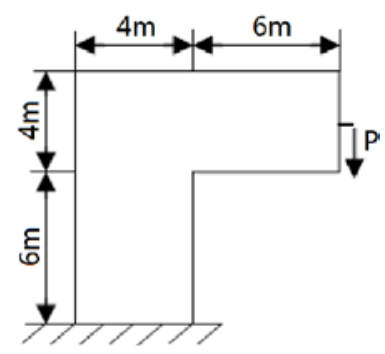

Fig.1. Dimensions of the design domain for a L column

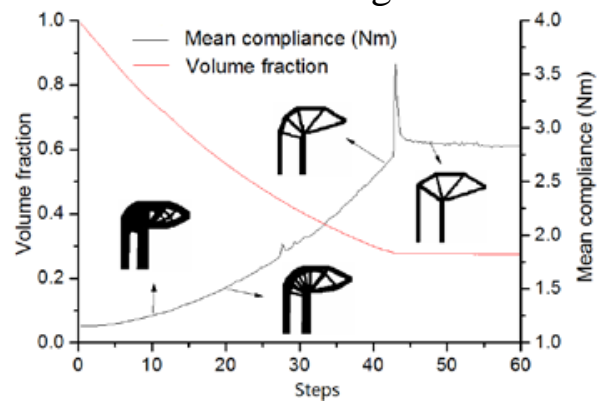

Fig.3. Evolution histories for the L column

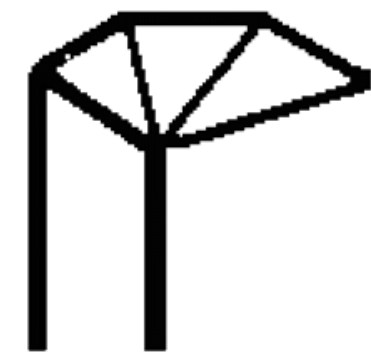

Fig.2.Optimal topology for the L column

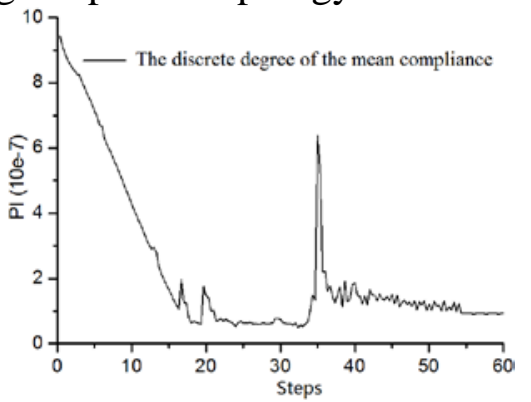

Fig.4. Evolution histories for the estimate Criterion

Fig. 2 shows the final reasonable topology shape, which is beneficial to the processing and manufacturing. And the material distribution is uniform, so the optimization results are satisfactory. Fig. 3 shows the evolution histories of the compliance and of the model decreases step by step until the objective volume is reached. It is clearly to see that the compliance exist big jump at the 45 steps as shown in Fig.3 and Fig.4. It is mainly because the connecting rod of the structure is changed from four to three, so the mechanism of force mechanism is changed in the process of deleting. And after that the evolution histories come to a reasonable and stable stage.

For the architectural design, some holes with certain size, such as doors and windows, needed in order to meet the need of the use function. For the topology design, those specific needs of the reserved area are defined as non-design elements. A wall with a door is given in Fig.5.

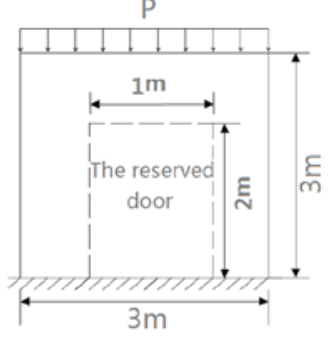

(a) The size required no less than the prescribed;

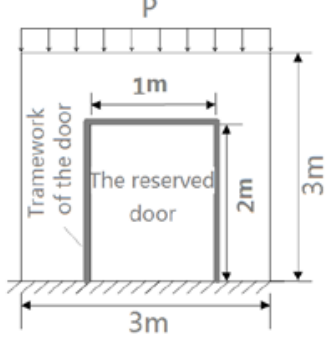

(b) the size required is exactly as the prescribed.

Fig.5. Dimensions of the design domain for a wall:
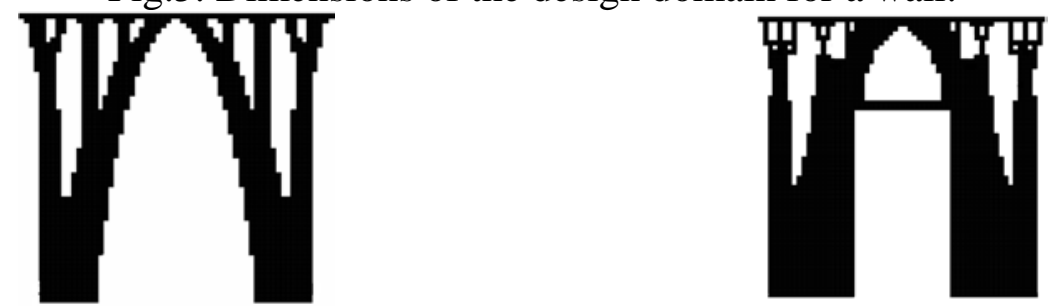

(a) The result for the size required no less than the prescribed;

(b)The result for the size required exactly as the prescribed.

Fig.6. Optimization results: 
A wall with different geometrical constraints door is given in Fig 5. And the functional requirements results shown in Fig. 6 are got by the method given in this paper. And both of them are beautiful and reasonable.

\section{Conclusion}

In this paper, the improved BESO optimization method is used to make the optimization process more reasonable and credible. The new criterion makes the discrete degree of data set judgment more reasonable. And it makes the objective function converges steadily; the accuracy of elemental sensitivity number is improved by its historical information.

Two examples are given to illustrate that the regional definition can be used for the specific function and beauty requirements structure, which expand the practical engineering application BESO method.

\section{Acknowledgement}

The authors wish to thank the financial support by the Science Funds from Educational Commission of Yun' nan Province, China (2016zzx005).

\section{References}

[1] Wang Xianjie, Zhang Xun'an, Lian Yeda. The multi-objective optimization under random loads[J]. Applied Mechanics and Materials, 2012. (166): 548 - 552.

[2] Huang X, Xie Y M, Burry M C. A new algorithm for bidirectional evolutionary structural optimization. JSME International Journal Series [J], 2006, 49(4):1091-1099.

[3] Xie Y M, Huang X, et al. Recent Developments of Evolutionary Structural Optimization and Bi-directional Evolutionary structural optimization [J]. Advances in Mechanics, 2011, 41(4): 462-471.

[4] Bin Xu. Integrated optimization of structure and control systems for interconnected building structures subjected to earthquake, Journal of Vibration and Control, 2014, 20(9):1318 1332

[5] Xianjie Wang, Zhang Xun'an. Computer program for directed structure topology optimization[J]. Acta Mechanica Solida Sinica,2015.4 (28): 431-440.

[6] Xianjie Wang, Zhang Xun'an. Concurrent optimization for structure topology and its periodic multiphase materials micro distribution[J].Chinese Journal of Computational Mechanic, 2014. 18 (45): 18078 - 18082.

[7] Huang X, Xie Y M. Evolutionary topology optimization of continuum structures methods and applications. Chichester[M]: John Wiley and Sons Ltd, 2010.

[8] Huang X, Xie Y M. Convergent and mesh-independent solutions for the bi-directional evolutionary structural optimization method. Finite Elements in Analysis and Design[J], 2007, 43: 1039-1049. 